\title{
Effect of peep on esophageal catheter optimal calibration volume and esophageal pressure measurements
}

\author{
F Magni $^{1 *}$, D Ceriani ${ }^{1}$, L Castagna ${ }^{2}$, C Ferrari ${ }^{1}$, A Zanella ${ }^{1}$, V Scaravilli ${ }^{1}$, SM Colombo ${ }^{1}$, M Laratta ${ }^{1}$, N Patroniti ${ }^{1,3}$ \\ From ESICM LIVES 2015 \\ Berlin, Germany. 3-7 October 2015
}

\begin{abstract}
Introduction
The use of esophageal balloon catheter to estimate pleural pressure has gained renewed popularity in recent years. Indeed, measurement of transpulmonary pressure may allow a more pathophysiological-based approach to ventilator strategy in ARDS patients. Nevertheless it is well known that esophageal balloon catheter derived parameters can be influenced by several patient-related or technical-related factors.
\end{abstract}

\section{Objectives}

To evaluate in-vivo the effect of positive end-expiratory pressure (PEEP) variations on esophageal catheter optimal calibration volume and measured esophageal pressure.

\section{Methods}

Experimental study in 8 (5 ARDS, 3 control) sedated, intubated, paralyzed and mechanically ventilated (volume-control) patients. Patients were monitored with esophageal balloon catheter (Cooper Surgical, Trumbull, CT USA).

Three PEEP groups were defined: low PEEP $_{\text {PE }}(8$ and 4 $\mathrm{cm} \mathrm{H}_{2} \mathrm{O}$ respectively in ARDS and control patients), medium $_{\text {PEEP }}\left(12\right.$ and $8 \mathrm{cmH}_{2} \mathrm{O}$ ) and high PEEP $_{\text {(16 }}$ and 12 $\mathrm{cmH}_{2} \mathrm{O}$ ).

During each PEEP level, we inflated the esophageal balloon with increasing amount of air (from 0.2 to $2 \mathrm{ml}$ ). For each injected volume, we performed an end-inspiratory occlusion maneuver followed by an occlusion test by applying manual chest compression during an end-expiratory airway occlusion maneuver. We measured the ratio between airway pressure variation and esophageal pressure variation $(\Delta \mathrm{Paw} / \Delta \mathrm{Pes}$ ratio) during the occlusion test, endexpiratory esophageal pressure (Pes,e), end-expiratory transpulmonary pressure $(\mathrm{Pl}, \mathrm{e})$, chest wall compliance $\left(\mathrm{Cpl} l_{\mathrm{CW}}\right)$, lung compliance $\left(\mathrm{Cpl}_{\mathrm{L}}\right)$, elastance-derived transpulmonary plateau pressure $(\Delta \mathrm{Pl}, \mathrm{i})$. The optimal calibration volume (defined as the injected volume at which $\triangle \mathrm{Paw} /$ $\triangle$ Pes ratio was closer to 1 ) was identified for each PEEP group $\left(\mathrm{VC}_{\mathrm{LPEEP}}\right.$ for low $_{\text {PEEP }}, \mathrm{VC}_{\mathrm{MPEEP}}$ for medium $\mathrm{PEEP}$, $\mathrm{VC}_{\text {HPEEP }}$ for high ${ }_{\text {PEEP }}$ ). Effect of PEEP on derived parameters was assessed by comparing at PEEP medium and high values obtained at the $\mathrm{VC}_{\mathrm{LPEEP}}$ against values obtained with the optimal VC at each PEEP.

\section{Results}

Optimal calibration volumes progressively raised with increasing PEEP $(0.95 \pm 0.14 \mathrm{ml}, 1.1 \pm 0,18 \mathrm{ml}, 1.22 \pm 0.2$ $\mathrm{ml}$ respectively for low ${ }_{\text {PEEP }}$, medium ${ }_{\text {PEEP }}$ and high ${ }_{\text {PEEP }} ; p<$ 0.001). See Figure 1. At high PEEP, Pes,e, $\mathrm{Cpl}_{\mathrm{L}}$ and $\Delta \mathrm{Pl}, \mathrm{i}$ were significantly higher while $\mathrm{Cpl}_{\mathrm{cw}}$ was significantly lower e when measured with $\mathrm{VC}_{\mathrm{HPEEP}}$ compared to $\mathrm{VC}_{\text {LPEEP. }}$

Partitioned respiratory system mechanic parameters are show in Table.

\section{Conclusions}

Esophageal catheter balloon calibration volume is affected by PEEP. Neglecting this effect may leads to errors in computing partitioned respiratory system mechanics. Catheter calibration should be checked after every change in PEEP.

${ }^{1}$ Università degli Studi di Milano Bicocca, Monza, Italy

Full list of author information is available at the end of the article 


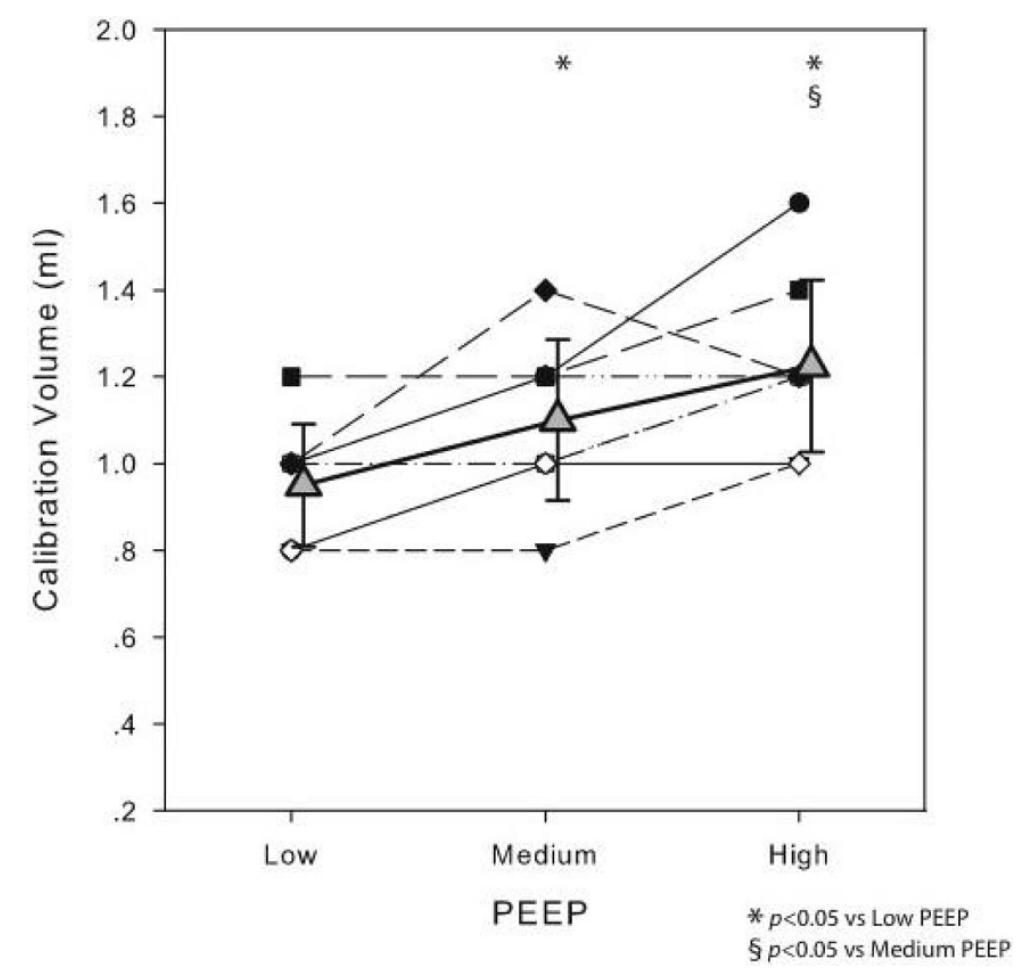

Figure 1 Optimal calibration volume and PEEP

\begin{tabular}{|c|c|c|c|}
\hline & & $V_{\text {LPEEP }}$ & $\mathrm{VC}_{\text {HPEEP }}$ \\
\hline & Pes,e $\left(\mathrm{cmH}_{2} \mathrm{O}\right)$ & $9.96 \pm 4.49$ & $10.76 \pm 4.35^{\star}$ \\
\hline & $\mathrm{Pl}, \mathrm{e}\left(\mathrm{cmH}_{2} \mathrm{O}\right)$ & $5.07 \pm 5.32$ & $4.32 \pm 5.02$ \\
\hline & $\mathrm{Cpl}_{\mathrm{L}}\left(\mathrm{ml} / \mathrm{cmH} \mathrm{cm}_{2} \mathrm{O}\right)$ & $66.1 \pm 39.37$ & $69.53 \pm 41.72^{*}$ \\
\hline & $\mathrm{Cpl}_{\mathrm{CW}}\left(\mathrm{ml} / \mathrm{cmH}_{2} \mathrm{O}\right)$ & $144(120 ; 170)$ & $124(104 ; 158)^{*}$ \\
\hline & $\Delta \mathrm{PI}, \mathrm{i}\left(\mathrm{cmH}_{2} \mathrm{O}\right)$ & $21.36 \pm 9.25$ & $20.35 \pm 8.67^{\star}$ \\
\hline
\end{tabular}

\section{Authors' details}

${ }^{1}$ Università degli Studi di Milano Bicocca, Monza, Italy. ${ }^{2}$ San Gerardo Hospital, Cardiac Intensive Care Unit, Monza, Italy. ${ }^{3}$ San Gerardo Hospital, Intensive Care Unit, Monza, Italy.

Published: 1 October 2015
doi:10.1186/2197-425X-3-S1-A1001

Cite this article as: Magni et al:: Effect of peep on esophageal catheter optimal calibration volume and esophageal pressure measurements. Intensive Care Medicine Experimental 2015 3(Suppl 1):A1001.

\section{References}

1. Talmor $D$, et al: Mechanical ventilation guided by esophageal pressure in acute lung injury. N Engl J Med 359:2095-2104.

2. Chiumello $D$, et al: Lung stress and strain during mechanical ventilation for acute respiratory distress syndrome. Am J Respir Crit Care Med 178:346-55. 